\title{
Cerclaje coracoclavicular y banda de tensión con sutura en el tratamiento de fracturas del tercio externo de la clavícula tipos II y V
}

\section{Coraco-clavicular loop and tension band suture in type II and type V distal-third clavicle fractures}

\author{
Jiménez I,* Muratore G,* Marcos-García A* \\ Hospital Universitario Insular de Gran Canaria.
}

RESUMEN. Se han descrito diferentes técnicas quirúrgicas para el tratamiento de las fracturas inestables del tercio distal de la clavícula. Las complicaciones asociadas y la necesidad de retirar los implantes siguen siendo motivo de preocupación. Proponemos una técnica quirúrgica que utiliza suturas de alta resistencia para restaurar la estabilidad vertical y horizontal en las fracturas del tercio distal de la clavícula de tipo II y V de la clasificación de Neer. Esta técnica se ha utilizado en tres casos; dos de tipo V y uno de tipo II. En todos se obtuvo la consolidación ósea y todos reanudaron sus actividades previas a la lesión, incluyendo actividad deportiva, a los seis meses de la cirugía. La técnica de cerclaje coracoclavicular y banda de tensión con sutura es un procedimiento sencillo que permite la estabilización vertical y horizontal de la fractura. Permite obtener buenos resultados clínicos y puede ser una alternativa costeefectiva eficaz en el tratamiento de estas lesiones aunque se requiere una serie más larga y un seguimiento a largo plazo para evaluar adecuadamente los resultados.

Palabras clave: Clavícula distal, coracoclavicular, fijación sutura, tercio externo, tratamiento.
ABSTRACT. Many surgical techniques have been used to address unstable distal third clavicle fractures. Complications and the need for hardware removal are still a concern. We propose a surgical technical using highstrength sutures to restore vertical and horizontal stability in Neer type II and Neer type V distal-third clavicle fractures. It has been used in three cases; two type V and one type II. In all cases, bone healing was achieved uneventfully and all patients resumed their pre-injury activities including sports at six-months postoperatively. The coraco-clavicular loop and tension band suture technique is a simple procedure that allows vertical and horizontal stabilization of the fracture. It achieves good clinical results and it may be a cost-effective alternative to other techniques although a longer series and long-term follow-up is required to adequately assess the results.

Keywords: Distal clavicle fracture, coracoclavicular fixation, lateral clavicle, suture technique.

* Departamento de Cirugía Ortopédica y Traumatología, Hospital Universitario Insular de Gran Canaria. Las Palmas de Gran Canaria, España.

ORCID:

Isidro Jiménez MD, FEBOT: 0000-0001-9922-5805.

Gustavo Muratore MD: 0000-0001-7707-1913.

Alberto Marcos-García MD: 0000-0003-0249-1280.

Correspondencia:

Dr. Isidro Jiménez

Avenida Marítima del Sur s/n, Las Palmas de Gran Canaria, C.P. 35016. Las Palmas, España,

E-mail: isidro_jimenez@hotmail.com

Recibido: 21-01-2021. Aceptado: 17-05-2021.

Citar como: Jiménez I, Muratore G, Marcos-García A. Cerclaje coracoclavicular y banda de tensión con sutura en el tratamiento de fracturas del tercio externo de la clavícula tipos II y V. Acta Ortop Mex. 2021; 35(2): 236-239. https://dx.doi.org/10.35366/101873 


\section{Introducción}

Las fracturas del tercio lateral o tercio distal de la clavícula representan 15-28\% de todas las fracturas de clavícula, pero constituyen hasta $45 \%$ de los casos de seudoartrosis sintomática que requieren tratamiento adicional. , $^{1,3}$

Las fracturas inestables del tercio lateral, clasificadas por Neer como tipo II y tipo $\mathrm{V},{ }^{4}$ representan un desafío único para la fijación quirúrgica debido al pequeño tamaño de los fragmentos óseos, la habitual conminución, la ruptura de los ligamentos coracoclaviculares y las grandes fuerzas deformantes en los fragmentos de la fractura. Se han descrito numerosas técnicas quirúrgicas para el tratamiento de estas fracturas, entre ellas la fijación transacromial con agujas de Kirschner, la fijación con tornillo o cerclaje coracoclavicular, sistemas de botón-sutura en diferentes configuraciones y diferentes diseños de placas. ${ }^{1,3}$

Muchas de estas técnicas se han asociado a complicaciones frecuentes, la incidencia de complicaciones después del tratamiento quirúrgico de estas lesiones es alta, oscilando entre 2.4 y $40 \%$ dependiendo de la técnica e incluyendo las relacionadas con el material que obliga a una nueva cirugía para retirarlo. ${ }^{1,5}$

Consideramos que la técnica ideal debería proporcionar estabilidad vertical y horizontal, ser sencilla de realizar y también ser costo-efectiva. Proponemos una técnica quirúrgica con suturas de alta resistencia que podría ser útil en el tratamiento quirúrgico de las fracturas inestables del tercio externo de la clavícula.

\section{Técnica quirúrgica}

La cirugía se realizó bajo anestesia general y en la posición de silla de playa en todos los casos, aunque se podría realizar en posición supina según la preferencia del cirujano. Se realizó la incisión cutánea siguiendo el borde anterior de la clavícula desde la articulación acromioclavicular

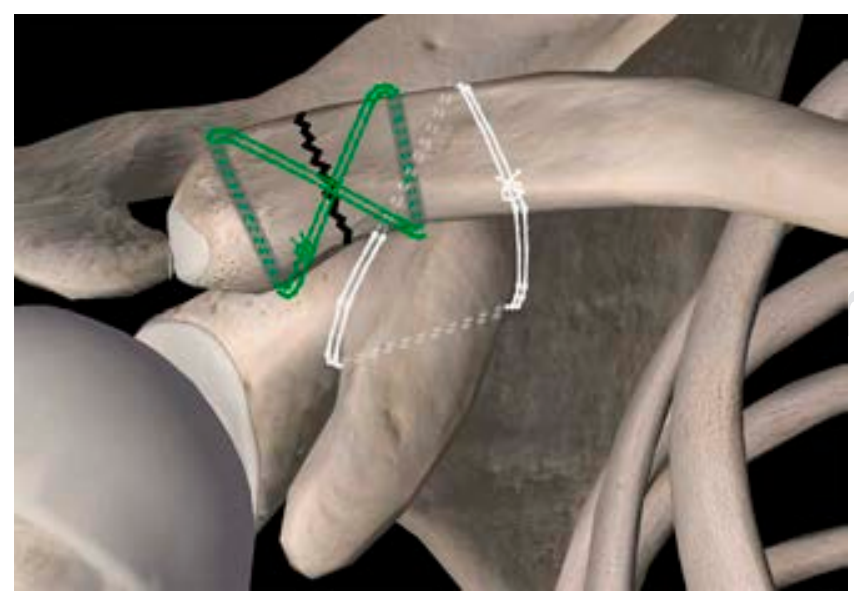

Figura 1: Esquema de la técnica quirúrgica. El bucle de sutura coracoclavicular (sutura blanca) permite lograr la estabilización vertical y la banda de tensión dorsal (sutura verde) asegura la estabilización horizontal.

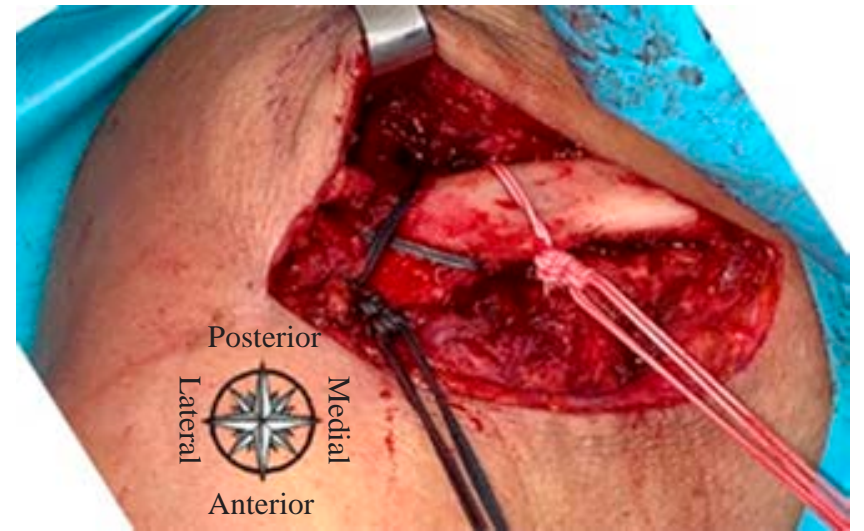

Figura 2: Imagen intraoperatoria en una mujer de 37 años (caso 3) con una fractura tipo II de Neer. La estabilización vertical se consigue mediante la sutura coracoclavicular (sutura blanca). La estabilización horizontal se obtiene creando una banda de tensión dorsal entre los fragmentos (sutura verde). Los nudos de sutura deben colocarse en la cara anterior de la clavícula para evitar problemas cutáneos.

(AC) hasta el nivel de la apófisis coracoides. La fractura se expuso mediante disección superióstica dejando la cápsula de la articulación AC intacta. Tras disecar de forma roma los márgenes medial y lateral de la apófisis coracoides, se pasó una sutura de polietileno tereftalato (PET) de número 5 (Smartloop ${ }^{\circledR}$, Fx Solutions, Viriat, Francia) en bucle por debajo de la base de la coracoides con ayuda de una pinza angulada tipo Satinsky. El extremo lateral de la sutura se pasó entonces alrededor del borde posterior de la clavícula y el extremo medial alrededor del borde anterior. La fractura se redujo elevando el hombro y aplicando presión hacia abajo en el fragmento medial de la clavícula, y las suturas se anudaron para mantener la reducción y controlar la estabilidad vertical.

A continuación, se realizó un agujero de dos milímetros en el fragmento medial de la clavícula en sentido anteroposterior y otro agujero en el fragmento distal adyacente a la articulación AC en el mismo eje. Se pasó una segunda sutura del número 5 en bucle a través de cada agujero formando una figura en ocho en la cara superior de la clavícula, solidarizando los fragmentos medial y lateral, se anudó la sutura creando una banda de tensión dorsal entre los fragmentos y consiguiendo la estabilidad horizontal (Figura 1). En los casos en los que el extremo distal de la clavícula se encuentra muy conminuto, el agujero del fragmento lateral podría hacerse en el acromion. Debe prestarse especial atención en ubicar los nudos de las suturas anteriores a la clavícula para evitar problemas cutáneos (Figura 2). No se utilizó drenaje aspirativo. Se suturó la fascia delto-trapecio-pectoral y se realizó sutura cutánea mediante técnica intradérmica.

Se utilizó un cabestrillo durante cuatro semanas permitiendo ejercicios de mano y codo. La terapia física para movilización asistida del hombro se inició a las cuatro semanas y los ejercicios activos del hombro se iniciaron a las seis semanas postoperatorias. 


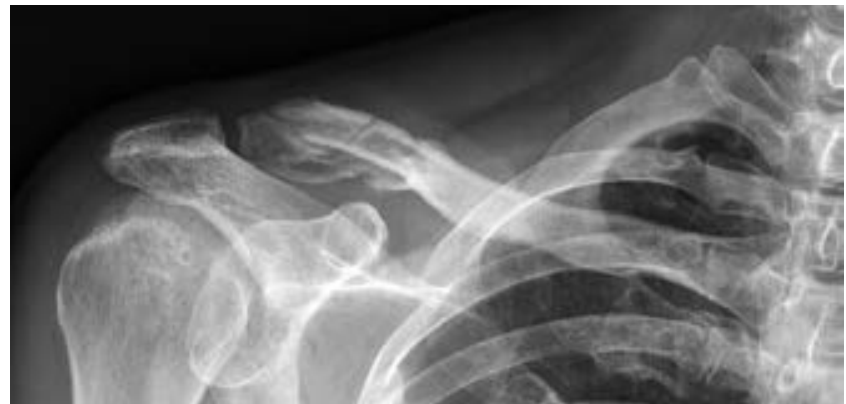

Figura 3: Aspecto radiológico de un hombre de 52 años (caso 1) con una fractura de tipo $\mathrm{V}$ de Neer a las cinco semanas postoperatorias.

\section{Resultados}

Esta técnica quirúrgica con suturas de alta resistencia se ha utilizado en tres casos; dos fracturas tipo $\mathrm{V}$ y uno tipo II. Dos hombres y una mujer con edad media de 39 años (rango: 27-52). El mecanismo lesional fue: caída fortuita desde propia altura en dos casos y caída durante la práctica deportiva en el caso restante. Todos los pacientes fueron operados durante la primera semana tras la lesión. La media de seguimiento postoperatorio fue de 14 meses (rango: 12-16).

En todos los casos se obtuvo la consolidación ósea sin incidencias (Figura 3) y todos los pacientes quedaron satisfechos con el resultado estético (Figura 4). A los seis meses de seguimiento postoperatorio, todos habían reanudado sus actividades previas a la lesión, incluida la actividad deportiva. Al año de la lesión, la puntuación media en el cuestionario de la American Shoulder and Elbow Surgeons (ASES) fue de 85.5 puntos (rango: 83.3-88.3) y la puntuación media del cuestionario Disabilities of the Arm, Shoulder and Hand (DASH) fue de 7.5 (rango: .4.5-11.4). No se observaron complicaciones y todos los pacientes refirieron encontrarse satisfechos con el resultado.

\section{Discusión}

Las fracturas inestables del tercio distal de la clavícula se asocian con altas tasas de seudoartrosis sintomática cuando se tratan de forma conservadora. Se han propuesto diferentes técnicas quirúrgicas, a pesar de lo cual las complicaciones asociadas y la necesidad de nuevos procedimientos, incluida la retirada del material implantando, siguen siendo motivo de preocupación. ${ }^{1,6}$

La estabilización coracoclavicular como tratamiento de la fractura del tercio lateral de la clavícula ha sido ampliamente reportada en la bibliografía, adaptándose para ello las técnicas inicialmente descritas para el tratamiento de las luxaciones AC. Estas técnicas han demostrado buenos resultados clínicos y radiográficos, pero la mayoría de ellas no permiten obtener una estabilidad en plano anteroposterior. ${ }^{1}$

Por otro lado, se ha publicado una técnica de osteosíntesis de las fracturas del tercio distal de la clavícula tipo II de Neer, utilizando suturas entre los fragmentos óseos reportando también buenos resultados clínicos, pero esta técnica por sí sola no aborda la inestabilidad cráneo-caudal. ${ }^{2,}$

Los principales beneficios de esta técnica de cerclaje coraco-clavicular y banda de tensión con sutura son: 1) permite la estabilización horizontal y vertical, por lo que puede ser útil en las fracturas de tipo II y tipo $\mathrm{V}$ y también en las luxaciones AC; 2) es técnicamente sencilla y no requiere implantes específicos; y 3) el uso de suturas es útil para evitar cirugías adicionales que habitualmente son necesarias para extraer los implantes utilizados con otras técnicas, por ello esta técnica es probablemente coste-efectiva, aunque no se realizó un análisis económico para comparar esta técnica con otras descritas previamente.

Un aspecto a destacar de esta técnica es que requiere que la apófisis coracoides esté íntegra, por lo que no se podría realizar en aquellos pacientes con una fractura asociada de la coracoides. Además, durante la propia cirugía podría producirse una fractura de la coracoides. Por ello, creemos que es aconsejable disponer de otra opción de osteosíntesis como las agujas de Kirschner transacromiales que podrían utilizarse si se produjera una fractura.

La estabilización horizontal requiere un agujero de dos milímetros en el fragmento distal adyacente a la articulación AC. En algunos casos, el fragmento lateral puede estar muy conminuto, lo que impide este paso de la técnica. En estos casos, dispondríamos de tres opciones: 1) realizar el agujero lateral en el acromion; 2) utilizar una sutura transósea a través de hueso esponjoso de la clavícula distal; y 3) pasar la sutura a través de la cápsula articular de la articulación AC.

Como la cobertura subcutánea sobre la clavícula suele ser delgada, una complicación frecuente en el tratamiento de las fracturas de clavícula son los problemas cutáneos. Para evitar esta complicación, debemos prestar atención a colocar los nudos de la sutura en el margen anterior de la clavícula y también realizar un cierre meticuloso de la fascia delto-trapecio-pectoral.

Este trabajo presenta limitaciones: se trata de una serie de casos pequeña; el período de seguimiento es probablemente corto para evaluar las complicaciones a largo plazo derivadas de la sutura utilizada; no se dispuso de un grupo control para realizar un estudio comparativo.

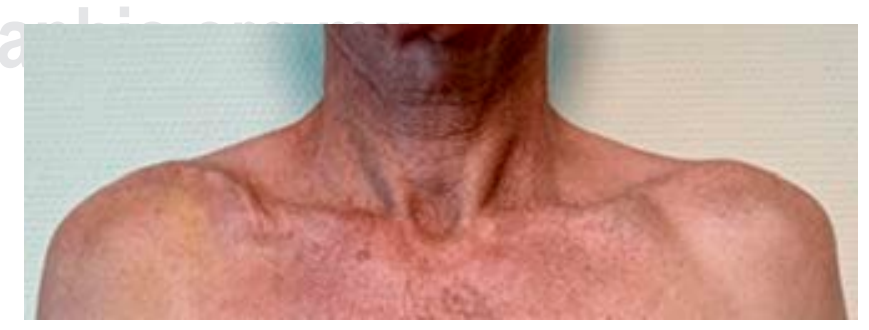

Figura 4: Aspecto clínico del caso 1 a las cinco semanas del postoperatorio. Obsérvese que ambas articulaciones acromioclaviculares están al mismo nivel. 


\section{Conclusión}

Con base en los resultados obtenidos, la técnica de cerclaje coracoclavicular y banda de tensión con sutura es un procedimiento sencillo que permite la estabilización vertical y horizontal de las fracturas inestables del tercio distal de la clavícula. Permite obtener buenos resultados clínicos y puede ser una alternativa costo-efectiva en comparación con otras técnicas aunque se requiere una serie más extensa y un seguimiento postoperatorio mayor para evaluar adecuadamente los resultados.

Referencias

1. Yagnik GP, Brady PC, Zimmerman JP, Jordan CJ, Porter DA. A biomechanical comparison of new techniques for distal clavicular fracture repair versus locked plating. J Shoulder Elbow Surg. 2019; 28: 982-8.

2. Badhe SP, Lawrence TM, Clark DI. Tension band suturing for the treatment of displaced type 2 lateral end clavicle fractures. Arch Orthop Trauma Surg. 2007; 127: 25-8.

3. Boonard M, Sumanont S, Arirachakaran A, Sikarinkul E, Ratanapongpean P, Kanchanatawan W, et al. Fixation method for treatment of unstable distal clavicle fracture: systematic review and network meta-analysis. Eur J Orthop Surg Traumatol. 2018; 28: 1065-78.
4. Neer CS 2nd. Fractures of the distal third of the clavicle. Clin Orthop Relat Res. 1968; 58: 43-50.

5. Rose MT, Noyes MP, Denard PJ. Arthroscopy-assisted treatment of displaced distal clavicle fractures utilizing curved buttons and suture tape with a single coracoclavicular tunnel. Tech Hand Up Extrem Surg. 2018; 22: 94-8.

6. Levy O. Simple, minimally invasive surgical technique for treatment of type 2 fractures of the distal clavicle. J Shoulder Elbow Surg. 2003; 12: $24-8$.

7. Oh JH, Kim SH, Lee JH, Shin SH, Gong HS. Treatment of distal clavicle fracture: a systematic review of treatment modalities in 425 fractures. Arch Orthop Trauma Surg. 2011; 131: 525-33.

Declaración de consentimiento informado: Se obtuvo el consentimiento informado por escrito de todos los sujetos antes de ser incluidos en el estudio.

Declaración de derechos humanos y animales: Todos los procedimientos se realizaron de acuerdo a los estándares éticos y la Declaración de Helsinki de 1975, revisada en 2008. Comité de ética: Nuestra institución no requiere aprobación del comité ético para el reporte de casos individuales o series de casos.

Conflicto de intereses: Los autores declaran que no tienen ningún conflicto de intereses. 